\title{
Differential atmospheric refraction in integral-field spectroscopy: Effects and correction
}

\author{
Atmospheric refraction in IFS \\ S. Arribas, E. Mediavilla, B. García-Lorenzo, C. del Burgo, and J.J. Fuensalida \\ Instituto de Astrofísica de Canarias, E-38200 La Laguna, Tenerife, Spain \\ e-mail: sam@ll.iac.es, emg@ll.iac.es, bgarcia@ll.iac.es, cburgo@ll.iac.es, and jjf@ll.iac.es
}

Received August 12; accepted November 27, 1998

\begin{abstract}
The basic concepts for determining the effect of differential atmospheric refraction on integral-field (twodimensional) spectroscopic observations are presented, and a method for correcting the spectra from this effect is given. The method is demonstrated with real data.
\end{abstract}

Key words: atmospheric effects - instrumentation: spectrographs - techniques: spectroscopic

\section{Introduction}

Integral-field spectroscopy (IFS) provides a spectrum simultaneously for each spatial sample of a two-dimensional field. This technique has merited much attention in recent years due to its advantages with respect to classical sequential 2-D spectroscopic techniques (e.g., longslit scans, Fabry-Perots) when studying relatively small extended objects. Several approaches to IFS have been developed, based in the use of fibres (e.g. Vanderriest \& Lemonier 1988; Barden \& Wade 1988; Arribas et al. 1991), microlenses (e.g. Bacon et al. 1995), micro-mirrors (e.g. Weitzel et al. 1996; Content 1998), or mixed solutions (e.g. Allington-Smith et al. 1997).

Most of the advantages of the IFS technique are direct consequences of the simultaneity when recording spatial and spectral information. The simultaneity not only implies a more efficient way of observing but, more importantly, it guarantees a great homogeneity in the data. In addition, IFS has other advantages. For instance, with IFS systems there is no need for an accurate centring of the object in the slit or to adapt the slit width (spectral resolution) to the seeing conditions; neither are the data

Send offprint requests to $:$ S. Arribas affected by "slit effects" when determining radial velocities (cf. Vanderriest 1995).

Here we want to demonstrate another relevant advantage of IFS. In many circunstances it is possible to determine and to correct in the spectra the effects of differential atmospheric refraction, using an a posteriori procedure. This is obviously important to preserve the spectrophotometric properties of the spectra without the use of atmospheric dispersion correctors (ADCs). Note that for longslit observations the presence of differential atmospheric refraction imposes strong restrictions which cannot be corrected by any means. Thus, the slit must be orientated along a direction defined by the parallactic angle (i.e. the spatial direction is predefined) if it is required to preserve the relative fluxes in the spectrum. If the slit is orientated at a desired position angle (no coincident with the one along which the differential atmosperic refraction takes place) special filters for the acquisition/guiding systems should be used to optimize the detection in a particular wavelength range, other spectral ranges being affected by light losses. To have a complete feel for these difficulties, we should note that the effects of differential atmospheric refraction are active, in the sense that they change with time. All these problems are strong drawbacks to obtain 2-D spectroscopic maps by scanning with a long slit. However, an approach using the a priori (theoretical) estimates of the shifts induced by differential atmospheric refraction to correct long slit data was proposed by Walsh \& Roy (1990).

In this paper we study the differential atmospheric refraction effects in IFS, and the basic concepts on which its correction should be performed. These steps will be illustrated with an example drawn from observational data. 

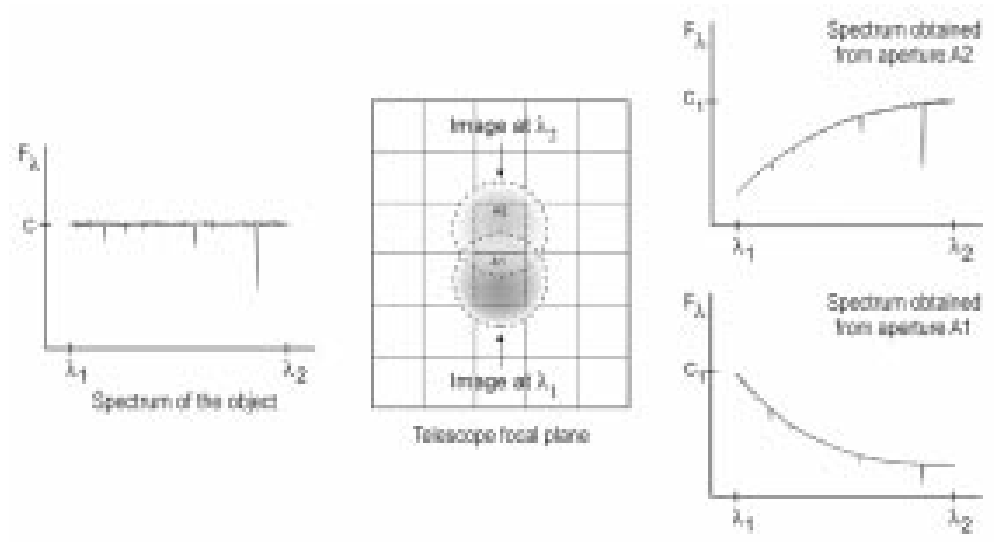

Fig. 1. Effects of differential atmospheric refraction on IFS. The spectra obtained from two different apertures differ between them and from the real one

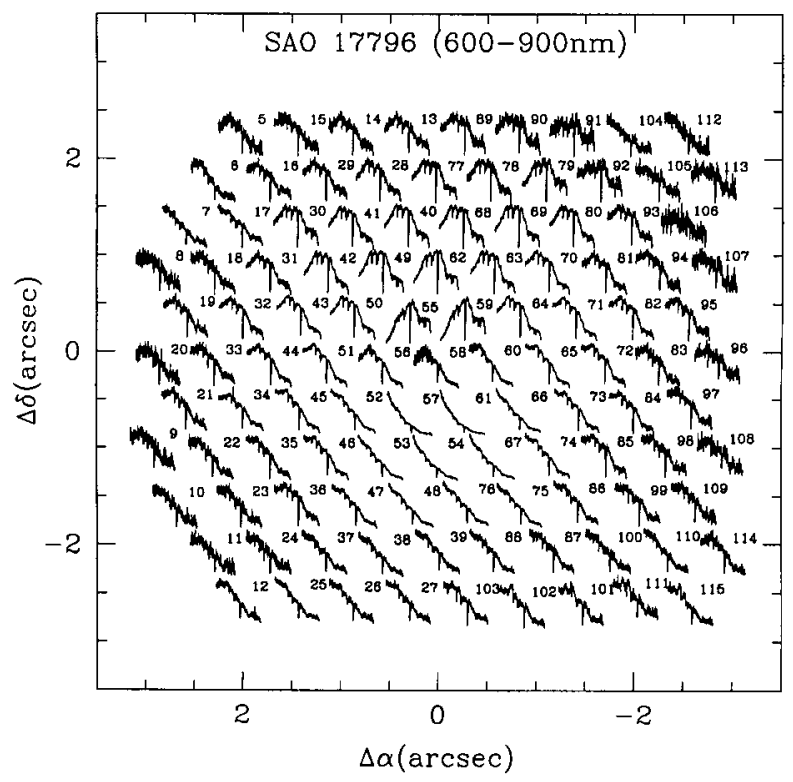

Fig. 2. Observed spectra from each aperture (fibre) of the fibre bundle corresponding to an exposure of the star SAO 17796. Each spectrum is plotted at its relative location in the telescope focal plane. The spectral range represented is $600-900 \mathrm{~nm}$

\section{Differential atmospheric refraction in IFS: Its effects and correction}

To illustrate the effects of differential atmospheric refraction (hereafter DAR) in IFS systems we are going to present a simplified case. Let us assume that the object is a star with a constant continuum, i.e. $F_{\lambda}($ continuum $)=$ const. (Fig. 1 left), and the parallactic angle is $0 \mathrm{deg}$ (i.e. the differential atmospheric refraction takes place along the $\mathrm{N}-\mathrm{S}$ direction). At the telescope focal plane, the star image is sampled by an array of square apertures.

In the absence of DAR the images of the star at all the wavelengths are coincident, and the spectra obtained from any aperture are basically the same (Obviously, the counts

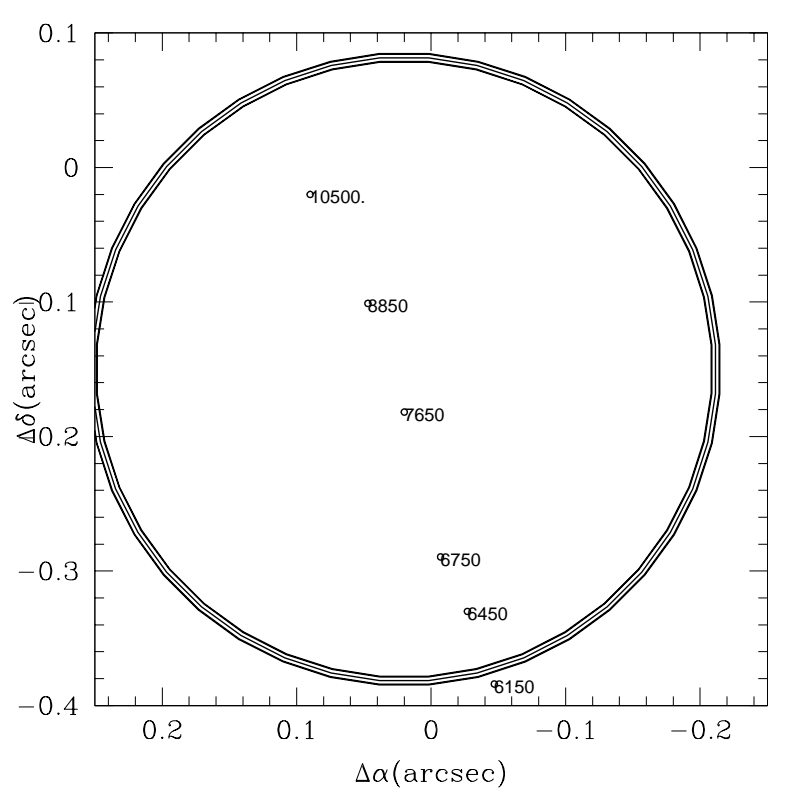

Fig. 3. Relative location of the maxima at different wavelengths (indicated in $\AA$ ). The circle represents the fibre core in adequate scale

and, therefore, the $\mathrm{S} / \mathrm{N}$ vary from aperture to aperture depending on their sampling of the seeing). However, when DAR is present the images of the star at different wavelengths are not in positional agreement. In our example they shifted along the N-S direction. Now each aperture gives a different spectrum. To illustrate this let us consider two individual apertures, $A_{1}$ and $A_{2}$, orientated along PA $0 \mathrm{deg}$ in such a way that the maximum of the star in $\lambda_{1}$ is at the centre of $A_{1}$, and the maximum of the star in $\lambda_{2}$ is at the centre of $A_{2}$ (see Fig. 1 centre). In such a situation aperture $A_{1}$ loses part of the light of the object's spectrum at wavelengths larger than $\lambda_{1}$. Similarly, aperture $A_{2}$ does not include part of the blue light of the star's spectrum. Consequently, the spectra inferred from $A_{1}$ and $A_{2}$ differ 


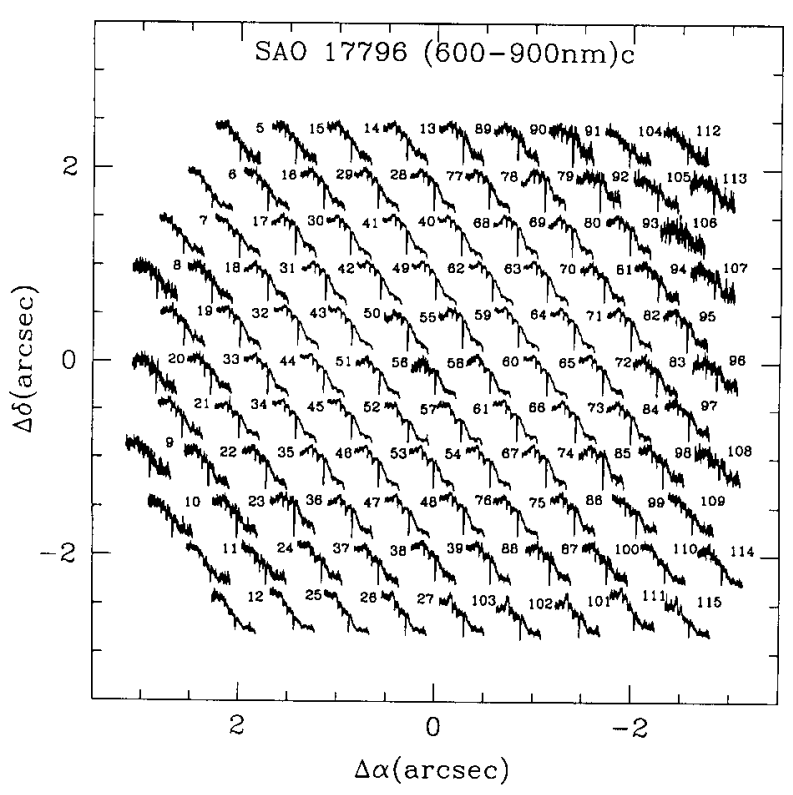

Fig. 4. Corrected spectra for each aperture (fibre) of the fibre bundle corresponding to an exposure of the star SAO 17796. Each spectrum is plotted at its relative location in the telescope focal plane. The spectral range represented is $600-900 \mathrm{~nm}$

between them and from the actual spectrum of the object (see Fig. 1). The fluxes obtained in this way at different wavelengths from the same aperture (fibre) spectrum are not directly comparable. In fact, in the previous example, the only comparable fluxes are those obtained in $\lambda_{1}$ from aperture $A_{1}\left(F_{\lambda_{1}}\left(A_{1}\right)\right)$, and in $\lambda_{2}$ from aperture $A_{2}$ $\left(F_{\lambda_{2}}\left(A_{2}\right)\right)$. In other words, if differential atmospheric refraction is present, the way to obtain the flux at a particu$\operatorname{lar} \lambda$ is to centre an aperture at the maximum of the star at this wavelength. If the complete spectrum is required, we should have centred as many apertures as spectral resolution elements, the actual spectrum being $F_{\lambda_{i}}=F_{\lambda_{i}}\left(A_{i}\right)$, where $F_{\lambda_{i}}\left(A_{i}\right)$ is the flux of an aperture centred on the maximum of the star in $\lambda_{i}$. Obviously it is not possible to have all these apertures on the focal plane simultaneously, especially if the number of spectral elements is in the order of 1000 . However, if the object is properly sampled, $F_{\lambda_{i}}\left(A_{i}\right)$ may be well determined by interpolation between $F_{\lambda_{i}}\left(A_{1}\right)$ and $F_{\lambda_{i}}\left(A_{2}\right)$. This is the basic method that we have applied for correcting for differential atmospheric refraction, and whose results are shown in the next section. Obviously, in a real case, the direction of differential atmospheric refraction and the distribution of the apertures are such that the interpolation must be done in two dimensions.

\section{Results}

\subsection{Observations}

The results presented here are based in observations taken on 1998 April 6 at the Observatorio del Roque de los
Muchachos (ORM) on the island of La Palma, using the INTEGRAL system (Arribas et al. 1998) at the $4.2-\mathrm{m}$ William Herschel Telescope (WHT).

We used the equalized optical-fibre bundle described by Arribas et al. (1998; hereafter AMF98), which at the telescope focal plane consists of a rectangular array of 115 optical fibres of $0.45^{\prime \prime}$ in diameter (see Fig. 1 of AMF98). The transmission of the fibres at the centre of the bundle are reduced in a controlled way in order to allow large dynamic range objects to be studied (i.e. equalized integral-field spectroscopy [EIFS]; see details in AMF98). This bundle was connected to the WYFFOS spectrograph (Bingham et al. 1994), which was equipped with a 316 -groove $\mathrm{mm}^{-1}$ grating, allowing the spectral range $5000-11000 \AA$ to be observed with a mean resolution of $11 \AA$ and a mean dispersion of about $6 \AA$ per pixel. With this configuration we took several exposures of the reference star SAO 17796. The atmospheric transmission was very poor with high clouds. However, the seeing (inferred from the data) was about 1 arcsec during the observations. The object was observed under a mean airmass of 1.28. The individual spectra were reduced following the standard procedure using IRAF, and some private codes.

In Fig. 2 we present the set of spectra after standard reduction obtained for an exposure of the star SAO 17796. Each of these spectra corresponds to the light collected by an aperture (fibre). With the fibre bundle we have sampled the PSF (the image of the star). In absence of DAR all the spectra should be identical (except for their $\mathrm{S} / \mathrm{N}$ ) but due to DAR there are large differences among the spectra taken at different positions (apertures) of the focal plane (see Fig. 2). Note, for instance, spectra 55 and 57. Clearly, spectrum 55 has partially lost the blue light, while the opposite occurs with spectrum 57 , which has lost part of the red.

\subsection{Differential atmospheric refraction characterization}

The first step for the correction of DAR effects is their characterization. For single objects this may be directly determined from the shifts among images generated at different wavelengths. For some extended objects point-like features in the observed field can be used. For instance, in Seyfert 1 galaxies, the location of the BLR emission from different lines (different $\lambda$ ) can be used to determine the DAR. However, in most cases no point-like features are available for extended objects, for which a displacement of the maximum at different wavelengths should not be directly interpreted as an effect of DAR. In these cases a simple model (e.g. Allen 1976) can be used to determine DAR effects.

In our simple case we have characterized DAR from the location of the maxima obtained from the maps generated at 6 different wavelengths. In Fig. 3 the distribution of the maxima of these maps is shown on sky coordinates. From this plot it is clear that DAR from $10500 \AA$ to 
$6150 \AA$ amounts to about 0.4 arcsec, the parallactic angle being $\sim 20 \mathrm{deg}$. This is in very agreement with the estimations by Fillipenko (1982) for an altitude of $2 \mathrm{~km}$, taking into account that the object was observed with a mean airmass of 1.28. Figure 3 also indicates the high accuracy when determining the maxima.

\subsection{Correction}

According to the concepts described in Sect. 2, we have determined the flux corrected from DAR for the aperture $j$ at the particular wavelength $\lambda_{i}$,

$F_{\lambda_{i}}^{\mathrm{c}}\left(\alpha_{j}, \delta_{j}\right)$,

following the transformation:

$F_{\lambda_{i}}^{\mathrm{c}}\left(\alpha_{j}, \delta_{j}\right)=F_{\lambda_{i}}\left(\alpha_{j}+\Delta \alpha_{i}, \delta_{j}+\Delta \delta_{i}\right)$,

where $\Delta \alpha_{i}$, and $\Delta \delta_{i}$ correspond to the shift due to DAR at the wavelength $\lambda_{i}$, taken as reference a given wavelength (10500 $\AA$, in our case). In our case these shifts are measured from the observational data but could be also calculated from the refractive index of the air.

For each aperture $j$, Eq. (2) is applied for all wavelengths, $\lambda_{i}$.

Note that $F_{\lambda_{i}}\left(\alpha_{j}+\Delta \alpha_{i}, \delta_{j}+\Delta \delta_{i}\right)$ is determined by interpolation of the observed values $F_{\lambda_{i}}\left(\alpha_{j}, \delta_{j}\right)$. Such an interpolation was done with the help of the NAG routine E01SAF. For each $\lambda$ this routine generates an interpolated two-dimensional surface $F(\alpha, \delta)$, which is continuous and has continuous first derivatives. The interpolant is then evaluated at the required position with the routine E01SBF.

In Fig. 4 we present the corrected spectra. We can see now that the large differences between the spectra obtained from the different apertures (Fig. 2) have disappeared.

\section{Summary}

Here we have demonstrated that using integral-field spectroscopic data, it is possible to determine and to correct in the spectra the effects of differential atmospheric refraction, using an a posteriori procedure. This implies an additional advantage with respect to classical sequential 2-D spectroscopic techniques, which are strongly limited by important DAR effects.
In particular, using an observational example, we have indicated how differential atmospheric refraction effects are determined in IFS, and the basic concepts according to which the spectra can be corrected for its effect.

Acknowledgements. We would like to thank Adolfo García his work in the development of the optical bundle. Thanks are also due to the referee, J.R. Walsh, for useful comments. We appreciate the help of Terry Mahoney and Gabriel Perez in the preparation of the manuscript.

The 4.2-m William Herschel Telescope is operated by the Isaac Newton Group of Telescopes at the Spanish Observatorio del Roque de los Muchachos of the Instituto de Astrofísica de Canarias. We thank all the staff at the Observatory for their kind support.

\section{References}

Allen C.W., 1976, Astrophysical Quantities. London, Athlone Press, pp. 124-125

Allington-Smith J.R., Content R.C., Haynes R., Lewis I.J., 1997, Proc. SPIE 2871, 1099

Arribas S., Mediavilla E., Rasilla J.L., 1991, ApJ 369, 270

Arribas S., Carter D., Cavaller L., et al., 1998, Proc. SPIE 3355,821

Arribas S., Mediavilla E., Fuensalida J.J., 1998, ApJ 505, L43 (AMF98)

Bacon R., Adam G., Baranne A., et al., 1995, A\&AS 113, 347

Barden S.C., Wade R.A., 1988, in Fiber Optics in Astronomy, Barben S. (ed.). ASP Conf. Ser. 3, 113

Bingham R.G., Gellatly D.W., Jenkins C.R., Worswick S.P., 1994, Proc. SPIE 2198, 56

Content R., 1998, Proc. SPIE, 3356 (in press)

Fillipenko A., 1982, PASP 94, 715

Vanderriest C., 1995, in 3D Optical Spectroscopic Methods in Astronomy, ASP Conf. Ser. 71, Comte G., Marcelin M. (eds.). San Francisco, ASP 209

Vanderriest C., Lemonier J.P., 1988, in Instrumentation for ground-based Astronomy, Robinson L. (ed.). New York, Springer-Verlag, p. 304

Walsh J.R., Roy J.-R., 1990, in Proc. 2nd ESO/ST-ECF Data Analysis Workshop, Baade D., Grosbol P.J. (eds.). ESO, Garching, p. 95

Weitzel L., Krabbe A., Kroker H., et al., 1996, A\&AS 119, 531 